\title{
Smear layer dissolution by peracetic acid of low concentration
}

\author{
De-Deus, G ; Souza, E M ; Marins, J R ; Reis, C ; Paciornik, S ; Zehnder, Matthias
}

\begin{abstract}
AIM: To test the effect of a noncaustic concentration of peracetic acid (PAA) in a standardized smear layer model. METHODOLOGY: The smear layer dissolution kinetics of $0.5 \%$ PAA on human dentine were compared to those of $2.25 \%$ PAA and $17 \%$ ethylenediaminetetraacetic acid (EDTA) solutions. Coronal dentine discs were prepared from six human maxillary molars. A standardized smear layer was produced on the pulpal side of each disc. The smear layer-covered surface was divided into three similar areas and then exposed to one of the three solutions tested. Co-site image sequences (around 40, $500 \times$ ) of the specific areas were obtained after four cumulative demineralisation times (15, 30, 60 and $180 \mathrm{~s}$ ). An image processing and analysis sequence measured sets of images, providing data of area fraction (AF, dentine-free area in \% of total analysis area). A general linear model for repeated measures was used to verify the influence of time and solution type over the change in AF from baseline $(\triangle \mathrm{AF})$. RESULTS: Overall, EDTA and $2.25 \%$ PAA produced higher $\triangle \mathrm{AF}$ values than the $0.5 \%$ PAA solution $(\mathrm{P}<0.05)$. No significant difference was observed in $\triangle \mathrm{AF}$ between $15 \mathrm{~s}$ and $30 \mathrm{~s}(\mathrm{P}>0.05)$. After $60 \mathrm{~s}$ of etching, all tested solutions produced similar $\triangle \mathrm{AF}(\mathrm{P}>0.05)$, whereas at $180 \mathrm{~s}, \Delta \mathrm{AF}$ of both EDTA and $2.25 \% \mathrm{PAA}$ continued to increase $(\mathrm{P}>0.05)$. Conclusions: After $60 \mathrm{~s}$ of contact, the $0.5 \% \mathrm{PAA}$ solution dissolved smear layer as well as 2.25\% PAA and 17\% EDTA. (c) 2011 International Endodontic Journal.
\end{abstract}

DOI: https://doi.org/10.1111/j.1365-2591.2010.01847.x

Posted at the Zurich Open Repository and Archive, University of Zurich

ZORA URL: https://doi.org/10.5167/uzh-58477

Journal Article

Accepted Version

Originally published at:

De-Deus, G; Souza, E M; Marins, J R; Reis, C; Paciornik, S; Zehnder, Matthias (2011). Smear layer dissolution by peracetic acid of low concentration. International Endodontic Journal, 44(6):485-490.

DOI: https://doi.org/10.1111/j.1365-2591.2010.01847.x 


\section{Abstract}

Aim To test the effect of a non-caustic concentration of peracetic acid (PAA) in a standardized smear layer model.

Methodology The smear layer dissolution kinetics of $0.5 \%$ PAA on human dentine were compared to those of $2.25 \%$ PAA and $17 \%$ EDTA solutions. Coronal dentine disks were prepared from six human maxillary molars. A standardized smear layer was produced on the pulpal side of each disk. The smear layer-covered surface was divided into three similar areas, and then exposed to one of the 3 solutions tested. Cosite image sequences (around 40,500x) of the specific areas were obtained after 4 cumulative demineralization times $(15 \mathrm{~s}, 30 \mathrm{~s}, 60 \mathrm{~s}$ and $180 \mathrm{~s})$. An image processing and analysis sequence measured sets of images, providing data of area fraction (AF, dentine-free area in \% of total analysis area). A general linear model for repeated measures was used to verify the influence of time and solution type over the change in AF from baseline $(\triangle \mathrm{AF})$.

Results Overall, EDTA and 2.25\% PAA produced higher $\triangle \mathrm{AF}$ values than the $0.5 \%$ PAA solution $(P<0.05)$. No significant difference was observed in $\triangle \mathrm{AF}$ between 15 $\mathrm{s}$ and $30 \mathrm{~s}(P>0.05)$. After $60 \mathrm{~s}$ of etching, all tested solutions produced similar $\Delta \mathrm{AF}$ $(P>0.05)$, whereas at $180 \mathrm{~s}, \triangle \mathrm{AF}$ of both EDTA and $2.25 \%$ PAA continued to increase $(P>0.05)$.

Conclusions After $60 \mathrm{~s}$ of contact, the $0.5 \%$ PAA solution dissolved smear layer with similar efficacy as $2.25 \%$ PAA and $17 \%$ EDTA did. 


\section{Introduction}

Despite a lack of strong evidence, removal of the smear layer that is created during mechanical root canal instrumentation (McComb \& Smith 1975) is considered to be an important treatment step (Torabinejad et al. 2002). This iatrogenic layer consists of a mixture of organic and inorganic debris (Sen et al. 1995). The organic portion is dissolved by sodium hypochlorite, the main endodontic irrigant (Zehnder 2006). To clear the inorganic portion of the smear layer a decalcifying agent is used, which can either be a chelator or an acid. Currently, all the products on the dental market sold to dissolve smear layer are based on ethylenediaminetetraacetic acid (EDTA) or citric acid. In theory, however, any biologically acceptable compound able to dissolve hydroxyapatite could be used for this specific purpose. For the sake of simplicity and to expedite root canal disinfection and debridement, it may be best to employ either a chemical that is compatible with sodium hypochlorite (Zehnder et al. 2005), or a counterpart that has a strong antimicrobial effect. Neither EDTA nor citric acid has strong antimicrobial properties (Zehnder et al. 2005, Bryce et al. 2009). Therefore, it has been proposed to use peracetic acid (PAA) instead of these "classical" decalcifying agents to dissolve the smear layer and concomitantly continue to disinfect the root canal system (Lottanti et al. 2009).

PAA solutions are among the strongest disinfectants known, with antibacterial, sporicidal, antifungal and antiviral properties (McDonnell \& Russell 1999). They have been used in the former German Democratic Republic as single endodontic irrigants (Kühlfluck \& Klammt 1980). Nowadays, PAA-related products are used mainly for veterinary purposes and for water treatment. In aqueous solution, peracetic acid is in equilibrium with hydrogen peroxide, acetic acid and acetylhydroperoxide. It is the acetic acid content that is probably responsible for the smear layer dissolution. Acetic acid forms complexes with calcium, which are easily soluble in water. As has been shown, a 2.25\% PAA solution has an effect on the smear layer and the root canal wall that is comparable to that of 17\% EDTA (Lottanti et al. 2009). However, 2.25\% PAA may be caustic when in contact with oral mucosa, and thus, it may be more advisable to use a lower concentration (Kühlfluck \& Klammt 1980). It is not known how the concentration of PAA affects its clearance of the smear layer. Furthermore, a direct comparison of the smear layer dissolution kinetics of PAA and EDTA has not 
been performed. Thus, the goal of the present investigation was to study the effect of the exposure time and concentration of PAA on removal of the smear layer. A standard $17 \%$ EDTA solution was used as a reference for comparison.

\section{Materials and Methods}

Specimen selection and dentine disk preparation

Six non-erupted third molars, recently extracted surgically, were kept in $0.2 \%$ sodium azide at $4^{\circ} \mathrm{C}$ for no longer than 7 days. The teeth were collected after the patients' informed consent had been obtained under a protocol reviewed and approved by the institutional Review Board (Ethics Committee).

Dentine disks approximately $3 \pm 0.3 \mathrm{~mm}$ thick were cut from the crown's middle third above the pulp chamber $(\mathrm{n}=6)$ ). A standard procedure (polishing with $\mathrm{SiC}$ paper $[200,300,400,600]$ grits and $3 \mu \mathrm{m}$ diamond paste) was employed on the pulpal surface of each disk, to prepare them for the experimental process and to produce a standardized smear layer (De-Deus et al. 2006, De-Deus et al. 2007).

\section{Co-site microscopy}

17\% EDTA solution was bought from a common commercial source (Formula \& Ação Ltda., São Paulo, SP, Brazil). PAA solutions were freshly prepared. According to the manufacturer (Kesla Pharma Wolfen GmbH, Greppin, Germany) the original commercial solution (Uterofertil, Kesla) contained 4.5\% (wt/vol) peracetic acid, 3.5\% acetic acid and 7.3\% hydrogen peroxide. It was diluted with bi-distilled water resulting in $2.25 \%$ and $0.5 \%$ PAA solutions.

To minimize the influence of the variability of human dentine when comparing different solutions, a single-tooth approach was followed (De-Deus et al. 2008). The central dentine area of each tooth was divided into 3 similar sections delimited by holes in an adhesive tape, $0.85 \mathrm{~mm}$ in diameter each. Each analysis area was exposed to $1 \mathrm{~mL}$ of the tested solutions for 4 cumulative experimental times (15, 30, 60 and $180 \mathrm{~s}$ ). After each experimental time, the demineralizing process was interrupted with $5 \mathrm{~mL}$ of bi-distilled water.

The experiment was performed with an Axioplan 2 Imaging motorized microscope (Carl Zeiss Vision Gmbh, Hallbergmoos, Germany) controlled by AxioVision 4.5 software (Carl Zeiss Vision). An Epiplan 50x HD objective lens was 
used coupled to a 1300 x 1030 pixels Axiocam HR digital camera (Carl Zeiss), resulting in a total magnification of approximately 500x, and a resolution of 0.21 $\mu \mathrm{m} /$ pixel.

The microscopic mosaic method was used to produce a single high-resolution image, composed of smaller images, which cover the full analysis area. Pre-defined mosaic settings controlled the microscopic motorized specimen stage to perform an automatic acquirement of 40 small images, at specific $x-y$ positions, so as to cover the whole analysis area. This procedure was followed for 4 cumulative demineralization times $(15,30,60$ and $180 \mathrm{~s})$. A previously developed image analysis routine (DeDeus et al. 2007, De-Deus et al. 2008a) was used to enhance image contrast, discriminate and measure open dentine tubules in each acquired image. The final product of the image analysis routine is the ratio between the total dentine-free area (open and eroded tubules) and the full analysis area - this ratio was termed area fraction (AF - \%). During the evaluation over time, each specimen served as its own control.

\section{Dentine mapping}

An automatic image analysis routine was developed to convert mean AF for each analysis area into false colours. A low-pass filter was used on the binary image, transforming it into a blurred grayscale image. Subsequently, a colour scale table was used to convert grayscale values into false colours (Fig. 1).

\section{Data presentation and analysis}

Considering that the AF of the full analysis area at the baseline (time point $=0$ s) were not similar among the experimental teeth due to the natural differences in the morphology of the dentine substrate, the obtained AF at each time point was deducted from the respective AF at the baseline. Consequently, the difference in AF compared to baseline $(\triangle \mathrm{AF})$ was used to express the effect of the solutions over the analysis area.

Considering the clustered nature of the data, a General Linear Model for repeated measures (GLMrep, SPSS 17.0, Inc., Chicago, IL, USA) was used to verify the influence of time and solutions over the $\triangle \mathrm{AF}$. Time was considered as the repeated factor, solutions the grouping factor. Bonferroni correction and Tukey tests were applied for multiple comparisons among time points and solutions respectively. 
Mauchly's Sphericity was used to verify the equality of the variances of the differences between levels of the repeated measures factor. The alpha-type error was set at 0.05 .

\section{Results}

The results of GLMrep indicated that sphericity was not assumed (Mauchly's sphericity, $P<0.05$ ), thus the Greenhouse-Geisser test was used to correct for violations of sphericity. The variation in time significantly influenced the $\triangle \mathrm{AF}$ of solutions (GLMrep, $P<0.05$ ). There was no significant difference in $\triangle \mathrm{AF}$ between $15 \mathrm{~s}$ and $30 \mathrm{~s}(P>0.05)$, whilst $\triangle \mathrm{AF}$ was significantly different from these time points at $60 \mathrm{~s}$ and $180 \mathrm{~s}(P<0.05)$. A significant difference was also found between time points $60 \mathrm{~s}$ and $180 \mathrm{~s}(P<0.05)$. The descriptive analysis of the data is displayed in Table 1 as $\triangle \mathrm{AF}$.

The type of solution significantly influenced the $\triangle \mathrm{AF}$ (GLMrep, $P<0.05$ ). Tukey post hoc test detected a significant difference in $\triangle \mathrm{AF}$ among the solutions in the following order: $0.05 \%$ PAA $<2.25 \%$ PAA $<17 \%$ EDTA $(P<0.05)$.

The interaction time-solutions was also significant (GLMrep, $P<0.05$ ), indicating that the $\triangle \mathrm{AF}$ differently varied along time according to the solution that was applied. From time point $15 \mathrm{~s}$ to time point $30 \mathrm{~s}$ solutions behaved differently $(P$ $<0.05)$. The same was seen for the time point $60 \mathrm{~s}$ to $180 \mathrm{~s}(P<0.05)$, whereas from time point $30 \mathrm{~s}$ to $60 \mathrm{~s}$ solutions had a similar effect on $\triangle \mathrm{AF}(P>0.05)$.

Qualitatively, all tested solutions produced increased demineralization over time and similar AF after $60 \mathrm{~s}$ of etching (Fig.1). Typical differences in dentine morphology over the experimental times are depicted in Fig. 2.

\section{Discussion}

The current study showed that PAA solutions, according to their concentration, can dissolve an experimental smear layer as quickly as a standard $17 \%$ EDTA solution does. After $60 \mathrm{~s}$ of contact, the 0.5\% PAA solution dissolved smear layer with similar efficacy as $2.25 \%$ PAA and $17 \%$ EDTA did.

The advantages and disadvantages of the current method have been discussed in detail earlier (De-Deus et al. 2007, De-Deus et al. 2008a, Reis et al. 2009, De-Deus 
et al. 2008b). The application of these results to the clinical situation is not straightforward. The goal of the present work was restricted to a direct comparison of the ability of PAA and EDTA to remove a standardized smear layer. One of the limitations of the current method is that the solutions were applied to a flat horizontal dentine surface and directly submitted the gravity force, different from the clinical situation, in which the contact between the demineralizing agent and the dentine surface is affected by the vertical position of the teeth and the intrinsic anatomical variability of the root canal system. On the other hand, several experimental parameters are better controlled in the current method, such as: the amount of available solution, contact surface area and contact time.

The current findings on PAA are in line with the quantitative results described by Reis and co-workers (Reis et al. 2009) in which a direct relation between chelator concentration and the increase in the AF was shown. Moreover, it should be noted that both PAA concentrations tested in the present study were able to remove the experimental smear layer to the same extend as EDTA after $60 \mathrm{~s}$. The use of lowconcentrate PAA solutions appears clinically attractive, as concentration is linked to tissue irritation. In a clinical trial, a solution containing $0.5 \%$ PAA did not irritate oral mucosa, whilst more concentrated solutions did (Kühlfluck \& Klammt 1980). Lottanti and co-workers (2009) have used scanning electron microscopy including energydispersive X-ray analysis to assess to which extent 17\% EDTA, 2.25\% PAA and 9\% etidronic acid removed the smear layer and demineralized the root canal wall. The authors concluded that all tested protocols resulted in clean canal walls. However, as was observed in root cross-sections, the effect on the root canal dentine differed between PAA and EDTA. This was in line with a study on simulated dentine caries, which showed a clear difference in mineral profiles obtained with acetic acid versus EDTA, with a gradual demineralization caused by acetic acid opposed to the total surface demineralization by EDTA (Kawasaki et al. 2000). Apparently, the high $\triangle \mathrm{AF}$ caused by the standard EDTA solution and $2.25 \%$ PAA at $180 \mathrm{~s}$ in this study is explained by erosion of dentine (Fig. 1 and 2). However, it must be cautioned that dentine erosion cannot be directly followed by the current methodology. Diluted EDTA solutions may have a different effect under the current conditions. Depending on the type of root filling and sealer, demineralization patterns may well be important. Total demineralization and a collapsed collagen network are generally not considered 
advantageous if the goal is to infiltrate the dentine with a resin to produce a so-called hybrid layer (García-Godoy et al. 2005, Tay et al. 2006). .

It is worthwhile underlining that PAA use could be clinically advantageous over the use EDTA if it could optimize the disinfection of the root canal space. Intracanal disinfection achieved with PAA in comparison to other decalcifying agents needs to be investigated in future studies. 


\section{References}

Bryce G, O'Donnell D, Ready D, Ng Y, Pratten J, Gulabivala K (2009) Contemporary root canal irrigants are able to disrupt and eradicate single- and dual-species biofilms. Journal of Endodontics 35, 1243-1248.

De-Deus G, Paciornik S, Pinho Mauricio M, Prioli R (2006) Real-time atomic force microscopy of root dentine during demineralization when subjected to chelating agents. International Endodontic Journal 39, 683-692.

De-Deus G, Reis CM, Fidel RA, Fidel SR, Paciornik S (2007) Co-site digital optical microscopy and image analysis: an approach to evaluate the process of dentine demineralization. International Endodontic Journal 40, 441-452.

De-Deus G, Zehnder M, Reis C, Fidel S, Fidel RA, Galan J Jr, Paciornik S (2008a) Longitudinal co-site optical microscopy study on the chelating ability of etidronate and EDTA using a comparative single-tooth model. Journal of Endodontics 34, 7175.

De-Deus G, Reis C, Fidel S, Fidel R, Paciornik S (2008b) Dentine demineralization when subjected to EDTA with or without various wetting agents: a co-site digital optical microscopy study. International Endodontic Journal 41, 279-287.

García-Godoy F, Loushine RJ, Itthagarun A, Weller RN, Murray PE, Feilzer AJ, Pashley DH, Tay FR (2005) Application of biologically-oriented dentin bonding principles to the use of endodontic irrigants. American Journal of Dentistry 18, 281290.

Kawasaki K, Ruben J, Tsuda H, Huysmans MC, Takagi O (2000) Relationship between mineral distributions in dentine lesions and subsequent remineralization in vitro. Caries Research 34, 395-403.

Kühlfluck I, Klammt J (1980) [Suitability of peracetic acid for root canal disinfection] (in German). Stomatologie DDR 30, 558-563. 
Lottanti S, Gautschi H, Sener B, Zehnder M (2009) Effects of ethylenediaminetetraacetic, etidronic and peracetic acid irrigation on human root dentine and the smear layer. International Endodontic Journal 42, 335-343.

McComb D, Smith DC (1975) A preliminary scanning electron microscopic study of root canals after endodontic procedures. Journal of Endodontics 1, 238-242.

McDonnell G, Russell AD (1999) Antiseptics and disinfectants: activity, action, and resistance. Clinical Microbiology Reviews 12, 147-179.

Reis C, De-Deus G, Leal F, Azevedo E, Coutinho-Filho T, Paciornik S (2008) Strong effect on dentin after the use of high concentrations of citric acid: an assessment with co-site optical microscopy and ESEM. Dental Materials 24, 1608-1615.

Sen BH, Wesselink PR, Türkün M (1995) The smear layer: a phenomenon in root canal therapy. International Endodontic Journal 28, 141-148.

Tay F, Pashley D, Loushine R, Doyle M, Gillespie W, Weller N, King N (2006) Ultrastructure of smear layer-covered intraradicular dentin after irrigation with BioPure MTAD. Journal of Endodonics 32, 218-221.

Torabinejad M, Handysides R, Khademi AA, Bakland LK (2002) Clinical implications of the smear layer in endodontics: a review. Oral Surgery Oral Medicine Oral Pathology Oral Radiology and Endodontis 94, 658-666.

Zehnder M (2006) Root canal irrigants. Journal of Endodontics 32, 389-398.

Zehnder M, Schmidlin P, Sener B, Waltimo T (2005) Chelation in root canal therapy reconsidered. Journal of Endodontics 31, 817-820. 


\section{Legends}

Table 1: Mean and standard deviations $( \pm \mathrm{SD})$ of $\triangle \mathrm{AF}$ in $\%$ for the tested solutions at each time point.

Figure 1: Dentine mapping highlighting the differences per tubule area fraction (AF) for each tested solution.

Figure 2: Surface changes of dentine regions during demineralization with each solution. The columns show the evolution of demineralization over time for $2 \%$ PAA, $0.5 \%$ PAA and $17 \%$ EDTA (from left). In each column, an image field at a specific $\mathrm{x}-$ $\mathrm{y}$ position of a specimen is shown for 4 cumulative demineralization times. The claim of high reproducibility of $x-y$ positions is confirmed by these figures as almost the exact same dentine features are visible for all times. 
Table 1 Mean and standard deviations $( \pm \mathrm{SD})$ of $\triangle \mathrm{AF}$ in $\%$ for the tested solutions at each time point.

\begin{tabular}{llllll}
\hline \multicolumn{5}{c}{ Time-point (s) } \\
\hline Solutions & 15 & 30 & 60 & 180 & Total \\
\hline $0.05 \%$ PAA & $0.19( \pm 0.40)$ & $0.32( \pm 0.44)$ & $0.71( \pm 0.87)$ & $0.80( \pm 0.88)$ & $0.51( \pm 0.73)$ \\
\hline $2.25 \%$ PAA & $0.30( \pm 0.37)$ & $0.51( \pm 0.65)$ & $0.80( \pm 0.92)$ & $2.30( \pm 2.33)$ & $0.98( \pm 1.52)$ \\
\hline EDTA & $0.64( \pm 0.81)$ & $0.9( \pm 0.67)$ & $1.06( \pm 0.84)$ & $2.18( \pm 1.60)$ & $1.20( \pm 1.20)$ \\
\hline
\end{tabular}




\section{然}
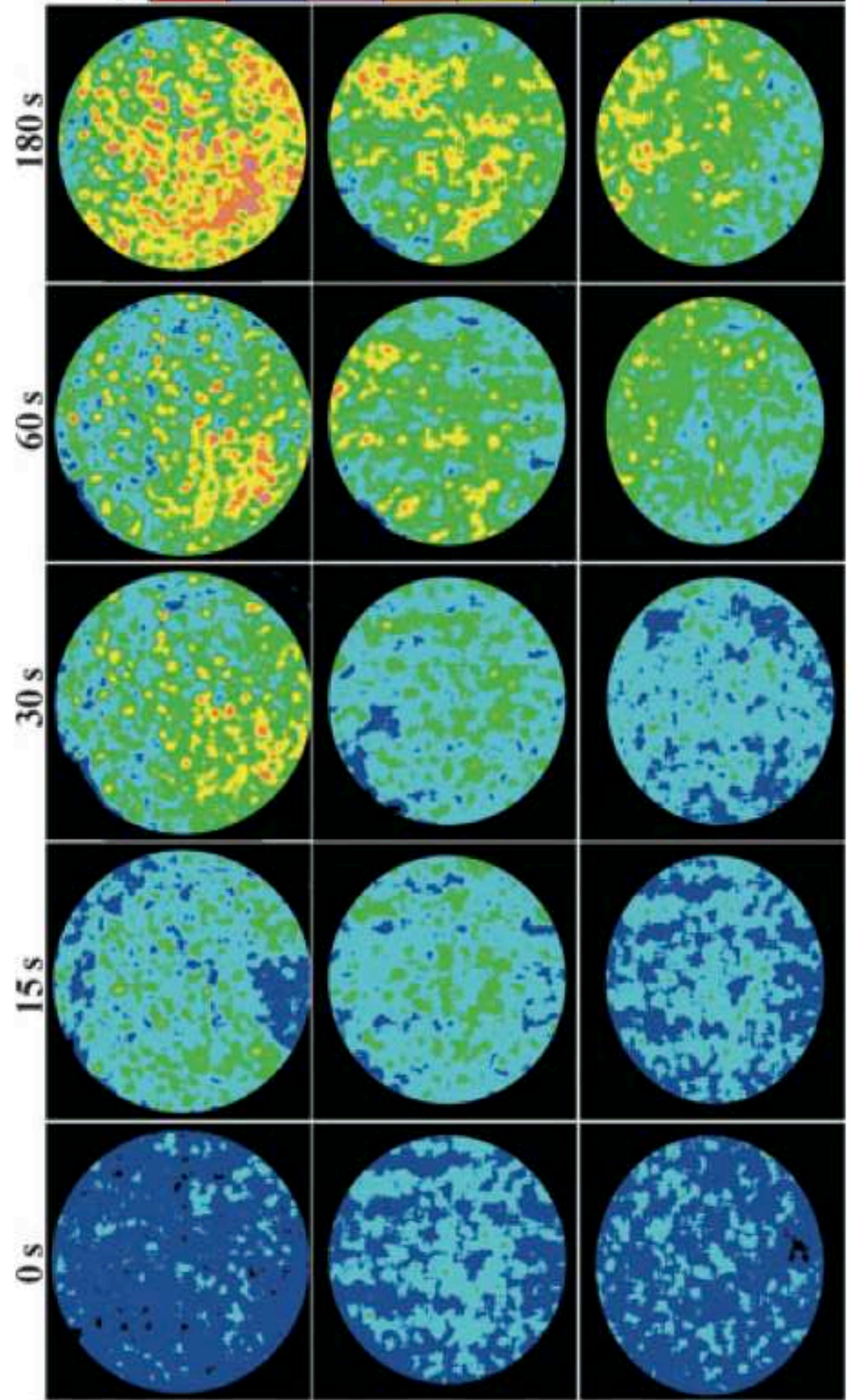


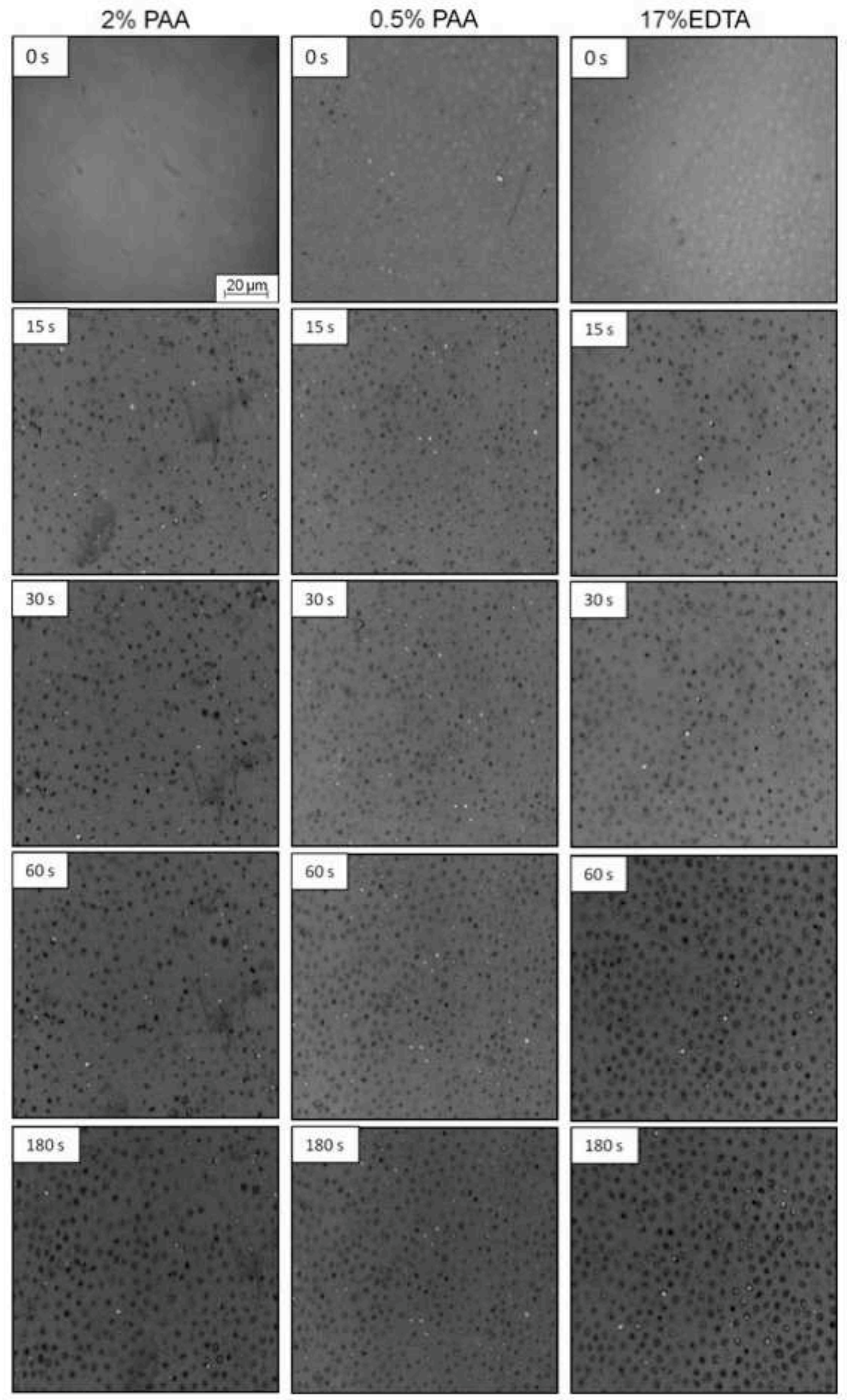

